Research Article

\title{
Effects of Three Traditional Chinese Fitness Exercises Combined with Antihypertensive Drugs on Patients with Essential Hypertension: A Systematic Review and Network Meta-Analysis of Randomized Controlled Trials
}

\author{
Lulu Dai $\mathbb{D}$, Yuerong Jiang $\mathbb{D}$, Peili Wang, and Keji Chen $\mathbb{D}$ \\ National Clinical Research Center for Chinese Medicine Cardiology, Xiyuan Hospital, \\ China Academy of Chinese Medical Sciences, Beijing 100091, China \\ Correspondence should be addressed to Yuerong Jiang; jiang_yuerong@163.com and Keji Chen; kjchenvip@163.com
}

Received 28 June 2021; Accepted 12 October 2021; Published 31 October 2021

Academic Editor: Woon-Man Kung

Copyright ( 2021 Lulu Dai et al. This is an open access article distributed under the Creative Commons Attribution License, which permits unrestricted use, distribution, and reproduction in any medium, provided the original work is properly cited.

\begin{abstract}
Objective. To compare the efficacy of three different traditional Chinese exercises (Tai Chi, Baduanjin, and Wuqinxi) combined with antihypertensive drugs (AHD) on patients with essential hypertension (EH). Method. Eight electronic databases were searched to identify randomized controlled trials (RCTs) comparing the effects of traditional Chinese fitness exercises combined with AHD and AHD alone. The analysis mainly consists of network meta-analysis (NMA) and pairwise meta-analysis. The Cochrane assessment tool was adopted to assess the risk of bias of included literatures. This study used STATA/SE 15.1 (StataCorp, 2017), R software (version 4.0.1), and Cochrane's Review Manager software (version 5.4) to conduct data analysis and figures generation. Results. A total of 30 RCTs were included in this study, of which 16 evaluated Tai Chi plus AHD versus AHD, 11 evaluated Baduanjin plus AHD versus AHD, and 3 evaluated Wuqinxi plus AHD versus AHD. No RCT compared directly among the three traditional Chinese fitness exercises. Pairwise meta-analysis showed that Tai Chi plus AHD was significantly superior to AHD alone in reducing systolic blood pressure (SBP) and diastolic blood pressure (DBP). BDJ plus AHD was statistically superior to AHD alone in reducing SBP, DBP, and endothelin (ET) and increasing nitric oxide (NO). NMA results indicated that Tai Chi plus AHD (WMD $-12.42 \mathrm{mmHg}, 95 \% \mathrm{CI}:-15.29$ to -9.55 ) and Baduanjin plus AHD (WMD $-7.03 \mathrm{mmHg}, 95 \% \mathrm{CI}$ : -9.80 to -4.26) were superior to AHD, and Tai Chi was more effective than other traditional exercises in lowering SBP, Tai Chi plus AHD (WMD -7.56 mmHg, 95\% CI: -10.15 to -4.96 ) and Baduanjin plus AHD (WMD $-4.51 \mathrm{mmHg}, 95 \%$ CI: -7.38 to -1.65 ) were superior to AHD in reducing DBP, Baduanjin plus AHD (WMD $4.26 \mu \mathrm{mol} / \mathrm{L}, 95 \% \mathrm{CI}$ : 2.68 to 5.83) was statistically superior to AHD in increasing NO, and Tai Chi plus AHD (WMD -7.64 pg/ml, 95\% CI: -10.46 to -4.83) and Baduanjin plus AHD (WMD $-9.23 \mathrm{pg} / \mathrm{ml}, 95 \% \mathrm{CI}:-10.85$ to -7.61 ) were superior to AHD in lowering ET. Conclusion. Compared with AHD alone, both Tai Chi plus AHD and Baduanjin plus AHD showed significant benefit in regulating SBP, DBP, and ET. Among the three traditional Chinese fitness exercises, Tai Chi may be the best as an adjunctive therapy for SBP reduction. These findings provided evidence for the therapeutic benefit of either Tai Chi or Baduanjin exercise as an adjunct therapy for patients with EH. Limited by the methodological quality and quantity of included studies, results need to be interpreted with caution, and it is necessary to carry out further high-quality RCTs on traditional Chinese fitness exercise-assisted treatment of EH in the future.
\end{abstract}

\section{Introduction}

The results of many cohort studies indicate that hypertension takes a leading role in the current global burden of cardiovascular disease and overall mortality $[1,2]$. Data from the Global Burden of Disease project shows that nearly 9.4 million deaths every year are due to raised blood pressure [3]. Therefore, prevention of the occurrence and progress of hypertension disorders is a current global priority public health problem [4]. Pharmacotherapy of hypertension is an important tool for treatment of hypertension [5]. However, there are adverse drug reactions caused by AHD, especially 
in the elderly, drug-drug interactions may also increase the burden related to drugs, and adverse reactions related to AHD can lead to the suspension of drug treatment [6-8]. Consequently, pharmacotherapy of hypertension combined with non-pharmacological method is very important; nonpharmacological method is a complementary treatment of drug therapy and delay the need for pharmacotherapy [9]. Exercise is an important non-pharmacological method to prevent, treat, and control elevated blood pressure. Patients can better regulate blood pressure by following an appropriate fitness exercise prescription, which can promote overall health and improve quality of life; low- to moderateintensity aerobic exercise is preferred for exercise therapy [10-14]. The 2017 Clinical Practice Guidelines for Hypertension recommends that adults diagnosed with hypertension or elevated blood pressure follow an organized exercise program to increase physical activity [15]. There have been some studies about exercise training practices on hypertension $[10-14,16]$, but comparatively little attention has been focused on exploring traditional Chinese fitness exercises as a complementary therapy for $\mathrm{EH}$, such as Tai Chi, Baduanjin, and Wuqinxi. While there have been some metaanalyses on the effects of traditional Chinese fitness exercises as an adjuvant therapy for $\mathrm{EH}$ [17-26], relatively scarce studies provide data from direct comparisons between these traditional Chinese fitness exercises. Therefore, to address this gap, we intend to perform a network meta-analysis (NMA) to compare the effects of three different traditional Chinese fitness exercises combined with AHD with AHD alone on patients with $\mathrm{EH}$. In contrast to traditional metaanalyses, which focus narrowly on a single-treatment comparison, a network meta-analysis is able to pool direct and indirect evidence, analyses all possible comparisons between all treatments for a disease, and assesses the relative merits of each treatment [27]. By means of NMA based on the frequentist framework, we could calculate surface under the cumulative ranking curve (SUCRA [28]) and the likelihood of being the best and the worst for each intervention to predict the curative effect ranking of each traditional Chinese fitness exercise, and provide direct information about the three types of traditional Chinese fitness exercises evidence.

\section{Methods and Analysis}

2.1. Search Strategy. Searches of the China Biology Medicine disc (CBM), China National Knowledge Infrastructure (CNKI), Wanfang Database (WANFANG), China Science and Technology Journal Database (CQVIP), Cochrane Central Register of Controlled Trials (CENTRAL), PubMed, EMBASE, and Web of Science were carried out for literatures published from journal inception to March 2021. There were no limitations in aspects of publication language or year in this search. The references in the relevant metaanalyses and systematic reviews and included literatures were reviewed carefully for relevant potential articles. PubMed search strategy is detailed in Table 1. For a complete search strategy of CENTRAL, PubMed, EMBASE, and Web of Science, be sure to read Supplementary Appendix 1.

\subsection{Eligibility Criteria}

2.2.1. Inclusion Criteria. Inclusion criteria were (1) RCTs comparing Tai Chi or Baduanjin or Wuqinxi plus AHD with AHD alone; (2) RCTs enrolling adults ( $\geq 18$ years) with EH (no restriction on gender, nation, or ethnic), according to the 2018 Chinese guidelines for the management of hypertension [29], the Seventh Report of the Joint National Committee on Prevention, Detection, Evaluation, and Treatment of High Blood Pressure (JNC 7) [30]; and (3) at least one of four interested outcome measures, which include SBP, DBP, NO, ET, and required to be documented in the literature.

2.2.2. Exclusion Criteria. Exclusion criteria were (1) nonrandomized controlled study; (2) participants with secondary hypertension or serious complications; (3) population or intervention not corresponding to our inclusion criteria; (4) no outcome measures of interest; (5) duplicated publications; (6) second publication of same trial; (7) conference abstract; (8) study protocol; (9) full text was not available; and (10) data cannot be extracted.

2.3. Study Selection. The retrieved literature records were managed by means of reference management software NoteExpress (version 3.2). We conducted the pilot selection of literature to make sure that the inter-rater reliability among assessors was high. Based on the inclusion and exclusion criteria, two independent researchers respectively conducted a detailed screening of titles and abstracts of reference records identified through database searching. All potential articles that meet the eligible criteria and controversial literatures were required for a full-text review. Arbitration will be carried out by the third researcher, who was responsible for resolving the confliction between the two researchers.

2.4. Data Extraction. Extraction of data of interest was separately conducted by two independent researchers after pilot extraction. The confliction between the two researchers would be resolved by the third researcher. The following data was what we need to extract: the first author of the research, publication year, diagnostic criteria for hypertension, level of blood pressure, sample, patient characteristics (age and sex), details of interventions, and outcome measures (SBP, DBP, $\mathrm{NO}$, and ET).

2.5. Risk of Bias Appraisal and GRADE Assessment. Based on Cochrane Handbook 5.1.0 [31], two independent researchers separately reviewed the included literatures to assess the risk of bias. The methodological quality of included studies was classified as having a low, unclear, or high risk of bias. Any confliction of opinions during the appraisal process was resolved by the third researcher or panel discussion. Assessment items included the following 7 items: (1) random sequence generation, (2) allocation concealment, (3) blinding of participants and personnel, (4) blinding of 
TABLe 1: Search strategy in PubMed.

\begin{tabular}{|c|c|}
\hline Search & Query \\
\hline$\# 1$ & $\begin{array}{l}\text { Search: (“Tai Ji”[Mesh]) OR (Tai-ji[Title/Abstract]) OR (Tai Chi[Title/Abstract]) OR (Chi, Tai[Title/Abstract]) OR (Tai Ji Quan } \\
\text { [Title/Abstract]) OR (Ji Quan, Tai[Title/Abstract]) OR (Quan, Tai Ji[Title/Abstract]) OR (Taiji[Title/Abstract]) OR (Taijiquan } \\
\text { [Title/Abstract]) OR (T’ai Chi[Title/Abstract]) OR (Tai Chi Chuan[Title/Abstract]) }\end{array}$ \\
\hline$\# 2$ & Search: (Wuqinxi[Title/Abstract]) OR (Baduanjin[Title/Abstract]) \\
\hline \#3 & $\begin{array}{c}\text { Search: ("Hypertension"[Mesh]) OR (Blood Pressure, High[Title/Abstract]) OR (Blood Pressures, High[Title/Abstract]) OR (High } \\
\text { Blood Pressure[Title/Abstract]) OR (High Blood Pressures[Title/Abstract]) }\end{array}$ \\
\hline \#4 & Search: randomized controlled trial[Publication Type] OR randomized[Title/Abstract] OR placebo[Title/Abstract] \\
\hline \#5 & \\
\hline \#6 & Search: \#3 AND \#4 AND \#5 \\
\hline
\end{tabular}

outcome data assessment, (5) incomplete outcome data, (6) selective outcome data reporting, and (7) other bias. The Grading of Recommendations Assessment, Development and Evaluation (GRADE) approach was applied to appraise the quality of the evidence behind the ranking of interventions from NMA [32].

\subsection{Statistical Analysis}

2.6.1. Pairwise Meta-Analyses. Cochrane’s Review Manager software (version 5.4) was used to analyze continuous data. We use the mean difference (MD) and 95\% CI for continuous variables. $I^{2}$ values were used to evaluate the statistical heterogeneity between the included studies. When there is no or low heterogeneity between studies $\left(I^{2}<25 \%\right)$, we used fixed-effects model to conduct the meta-analysis. If there is substantial heterogeneity $\left(25 \%<I^{2}<95 \%\right)$ and clinical heterogeneity was considered acceptable, we used random-effects model to conduct the meta-analysis. When the statistical heterogeneity is particularly large $\left(I^{2}>95 \%\right)$ or clinical heterogeneity is particularly significant, quantitative data were not pooled.

2.6.2. Network Meta-Analyses. This study used STATA/SE 15.1 (StataCorp, 2017) and $\mathrm{R}$ software (version 4.0.1) to conduct data analysis and figures generation. We used WMD and their associated 95\% CIs to summarize results. We took into account the existence of heterogeneity among different RCTs; thus, the random-effects model was selected to combine effect sizes in this network meta-analysis. We used the node-splitting model to assess inconsistency between direct and indirect comparisons. The bias in publication and small-scale study effects were evaluated with comparison-adjusted funnel plots, which were generated using "netfunnel" command. The network geometry of three different traditional Chinese fitness exercises was shown and described with network evidence plots, which were generated using "networkplot" command. We calculated the SUCRA and likelihood of being the best and the worst for each intervention to predict the curative effect ranking of each traditional Chinese fitness exercise. The significance level for all data analyses of this network meta-analysis was predetermined at 0.05 .

\section{Results}

3.1. Results of Study Selection. Initially, 488 records were identified through database searching. Then, duplicates were removed, and 255 records remained. 159 records were excluded after screening titles and abstracts. 96 studies were eligible for full-text screening. Finally, 30 studies met our inclusion criteria [26, 33-61]. The detailed selection process is illustrated in Figure 1.

3.2. Characteristics of Included Studies. Table 2 presents and describes the characteristics of included RCTs. This study involved 30 RCTs, 2160 participants with EH. All included RCTs were based on AHD-controlled two-arm studies. The years of publication of included RCTs were from 2006 to 2021. We included following three traditional Chinese fitness exercise types in our NMA: Tai Chi, Baduanjin, and Wuqinxi. 16 RCTs were Tai Chi interventions $[26,33,34,37,40,47-51,54-58,61], 11$ RCTs were Baduanjin

interventions $[35,36,38,39,41,42,44,46,52,59,60]$, and the other 3 RCTs were Wuqinxi interventions $[43,45,53]$. The length of interventions ranged from 8 weeks to 5 years ( 260 weeks), most of which were 12 or 24 weeks.

3.3. Methodological Quality. Figures 2 and 3 present the risk of bias assessment of included RCTs. The overall methodological quality of included studies was low. In terms of random sequence generation, 13 $[35,36,39,41,42,45,48,49,52,57,58,60,61]$ RCTs were judged as low risk and 17 $[26,33,34,37,38,40,43,44,46,47,50,51,53-56,59]$ were unclear risk. Twenty-eight [26, 34-60] RCTs were judged as unclear risk and $2[33,61]$ were judged as low risk in adequate allocation concealment. In terms of participants and personnel blinding, 27 [26, 34-37, 39-60] RCTs were judged as unclear risk and 2 $[33,61]$ showed low risk and 1 [38] was judged as high risk. In terms of outcome assessment blinding, 28 $[26,33-37,39-60]$ RCTs were judged as unclear risk and 2 [38, 61] were low risk. Twenty-nine [26, 33-52, 54-61] RCTs were judged as low risk and only 1 [53] was unclear risk in incomplete outcome data. In terms of selective 


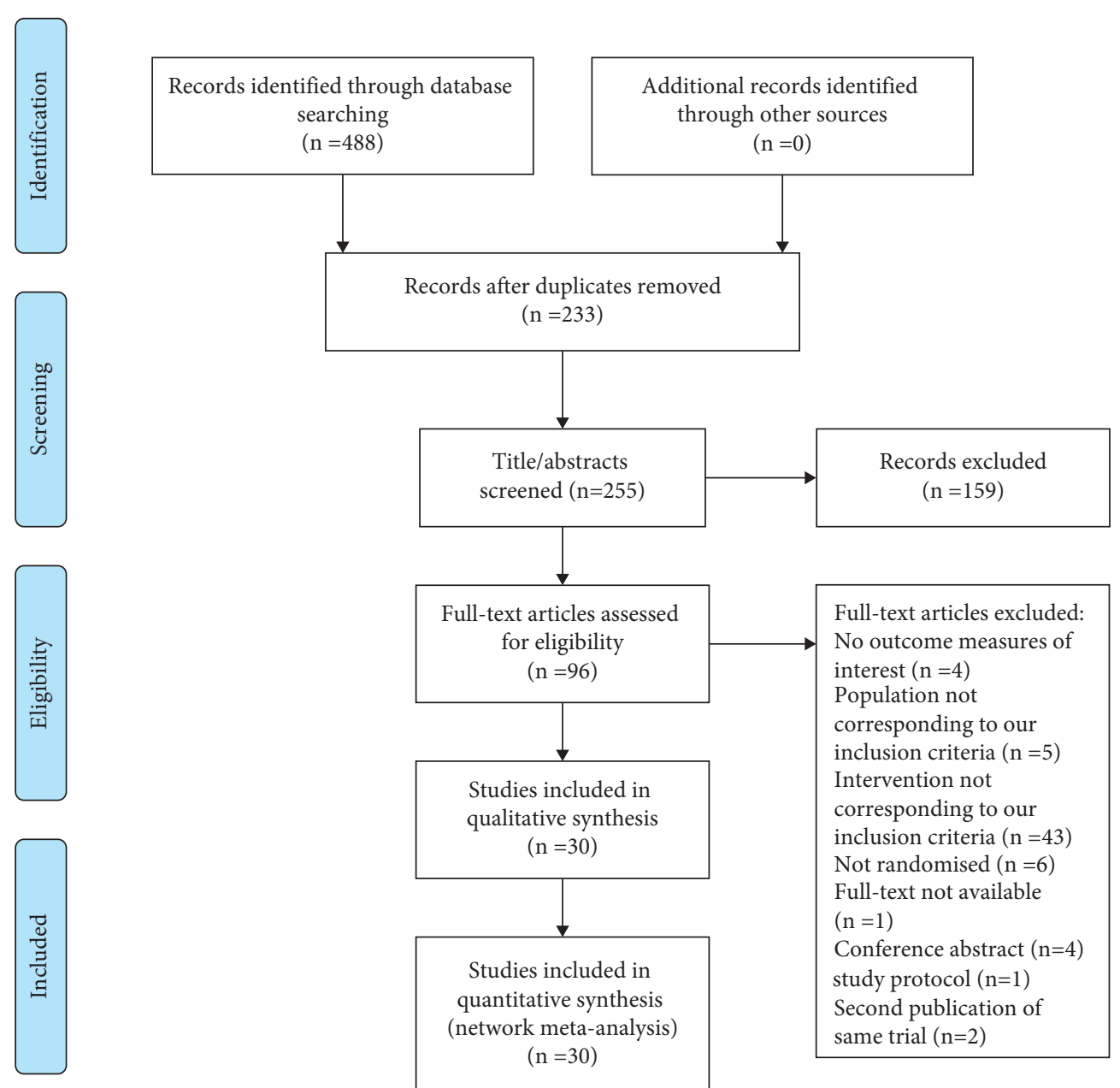

FIGURE 1: Flow diagram of literature identification and selection.

reporting, 3 [35, 41, 58] studies were judged as unclear risk, and 27 [26, 33, 34, 36-40, 42-57, 59-61] were low risks. In terms of other biases, 12 $[26,33,38,39,42,43,46,47,52,53,55,60]$ studies were judged as low risk and 17 [34-37, 40, 41, 44, 45, 48-51, 54, 56-59] were unclear risk and 1 [61] was high risk.

3.4. Results of Pairwise Meta-Analysis. Table 3 and Supplementary Appendix 2 show the results of pairwise meta-analysis. Tai Chi plus AHD was significantly superior to AHD alone in reducing SBP and DBP. BDJ plus AHD was statistically superior to AHD alone in reducing SBP, DBP, and ET and increasing NO. There were no statistically significant differences in other results.

\subsection{Results of Network Meta-Analysis}

3.5.1. Assessment of Inconsistency. Lack of direct comparison resulted in no closed loop between different interventions, and all the included studies were 2-arm trials, so there was no need for inconsistency testing.
3.5.2. Publication Bias. The bias in publication and smallscale study effects are illustrated in Figure 4. Funnel plots for the SBP, NO, and ET network were roughly symmetrical, indicating that there was no apparent bias of publication due to small-scale study effects. The asymmetrical distribution of the funnel plots for the DBP network indicated that there was a possibility of bias of publication due to small-scale study effects.

3.5.3. SBP Outcome. SBP was assessed in 29 trials $(2100$ participants). The network evidence plot for SBP is shown in Figure 5. In SBP reduction, Tai Chi plus AHD (WMD $-12.42 \mathrm{mmHg}$, 95\% CI: -15.29 to -9.55$)$ and Baduanjin plus AHD (WMD $-7.03 \mathrm{mmHg}, 95 \% \mathrm{CI}$ : -9.80 to -4.26 ) were superior to AHD alone, Baduanjin plus AHD (WMD $5.38 \mathrm{mmHg}$, 95\% CI: 1.39 to 9.37 ) and Wuqinxi plus AHD (WMD $8.26 \mathrm{mmHg}, 95 \% \mathrm{CI}: 1.66$ to 14.85 ) was statistically inferior to Tai Chi plus AHD; there was no statistically significant difference in other results (see Figure 6 and Table 4). According to SUCRA, the interventions to reduce SBP are ranked in probability (see Table 4 and Figure 7): $\mathrm{TC}+\mathrm{AHD} \quad(99.5 \%)>\mathrm{BDJ}+\mathrm{AHD} \quad(60.2 \%)>\mathrm{WQX}+\mathrm{AHD}$ 
TABLE 2: Characteristic of included studies.

\begin{tabular}{|c|c|c|c|c|c|c|c|c|}
\hline Author & $\begin{array}{c}\text { Diagnosis } \\
\text { criteria }\end{array}$ & level of blood pressure & $\begin{array}{l}\text { Sample } \\
\text { N/M/F }\end{array}$ & $\begin{array}{c}\text { Mean age } \\
\text { (years) }\end{array}$ & Interventions & $\begin{array}{c}\text { Exercise } \\
\text { prescription }\end{array}$ & $\begin{array}{c}\text { Length of } \\
\text { intervention } \\
\text { (weeks) }\end{array}$ & $\begin{array}{l}\text { Outcome } \\
\text { measures }\end{array}$ \\
\hline $\begin{array}{l}\text { Ma, } \\
2018\end{array}$ & NA & NA & $55 /-/-$ & $69 \pm 9.37$ & $\mathrm{TC}+\mathrm{AHD}$ & $\begin{array}{c}3 \sim 5 \text { times/week, } \\
\text { at least } 60 \text { mins/ } \\
\text { session }\end{array}$ & 24 & SBP; DBP \\
\hline $\begin{array}{l}\text { Chen, } \\
2013\end{array}$ & NA & NA & $\begin{array}{l}58 /-/- \\
50 /-/- \\
18 /-/-\end{array}$ & $30 \sim 82$ & $\begin{array}{c}\text { AHD } \\
\text { TC+ AHD } \\
\text { AHD }\end{array}$ & $\begin{array}{l}6 \text { times/week, } \\
30 \text { mins/session }\end{array}$ & 12 & SBP; DBP \\
\hline $\begin{array}{l}\text { Chen, } \\
2016\end{array}$ & $\begin{array}{c}\text { CGMH- } \\
2010\end{array}$ & NA & $\begin{array}{c}18 /-/- \\
28 / 15 / 13\end{array}$ & $69.98 \pm 3.11$ & $\mathrm{BDJ}+\mathrm{AHD}$ & $\begin{array}{l}5 \text { times/week, } \\
60 \text { mins/session }\end{array}$ & 12 & SBP; DBP \\
\hline $\begin{array}{l}\text { Chen, } \\
2013\end{array}$ & $\begin{array}{l}\text { CGMH- } \\
2005\end{array}$ & EH1 & $\begin{array}{l}28 / 14 / 14 \\
27 / 13 / 14 \\
28 / 16 / 12\end{array}$ & $\begin{array}{l}70.29 \pm 1.77 \\
69.23 \pm 3.72 \\
70.06 \pm 3.51\end{array}$ & $\begin{array}{c}\text { AHD } \\
\text { BDJ + AHD } \\
\text { AHD }\end{array}$ & $\begin{array}{c}\text { 5times/week, } \\
30 \text { mins/session }\end{array}$ & 12 & $\begin{array}{l}\text { SBP; DBP; } \\
\text { NO; ET }\end{array}$ \\
\hline $\begin{array}{l}\text { Chen, } \\
2006\end{array}$ & $\begin{array}{l}\text { CGMH- } \\
2005\end{array}$ & $\mathrm{EH} 2,3$ & $\begin{array}{c}2020 / 9 / \\
11 \\
20 / 13 / 7\end{array}$ & $\begin{array}{l}64.3 \\
60.7\end{array}$ & $\begin{array}{c}\mathrm{TC}+\mathrm{AHD} \\
\mathrm{AHD}\end{array}$ & $\begin{array}{c}1 \text { time/day, } \\
40 \text { mins/session }\end{array}$ & 10 & $\begin{array}{l}\text { SBP; DBP; } \\
\quad \text { NO }\end{array}$ \\
\hline $\begin{array}{l}\text { Dong, } \\
2020\end{array}$ & $\begin{array}{l}\text { CGMH- } \\
2005\end{array}$ & NA & $\begin{array}{l}21 /-/- \\
21 /-/-\end{array}$ & $30 \sim 65$ & $\begin{array}{c}\mathrm{BDJ}+\mathrm{AHD} \\
\mathrm{AHD}\end{array}$ & $\begin{array}{l}5 \text { times/week, } \\
60 \text { mins/session }\end{array}$ & 16 & $\begin{array}{l}\text { SBP; DBP; } \\
\text { ET }\end{array}$ \\
\hline $\begin{array}{l}\text { Fan, } \\
2021\end{array}$ & $\begin{array}{c}\text { CGMH- } \\
2018\end{array}$ & $\mathrm{EH} 1,2$ & $\begin{array}{l}38 / 21 / 17 \\
38 / 19 / 19\end{array}$ & $\begin{array}{l}71.87 \pm 0.76 \\
71.95 \pm 0.97\end{array}$ & $\begin{array}{c}\mathrm{BDJ}+\mathrm{AHD} \\
\text { AHD }\end{array}$ & $\begin{array}{c}5 \text { days/week, } \\
30 \text { mins/session }\end{array}$ & $\begin{array}{l}12 \\
12\end{array}$ & SBP; DBP \\
\hline $\begin{array}{l}\text { Han, } \\
2010\end{array}$ & $\begin{array}{c}1999 \\
\text { WHO-ISH }\end{array}$ & $\mathrm{EH} 1,2$ & $30 /-1-$ & $62.12 \pm 10.51$ & $\mathrm{TC}+\mathrm{AHD}$ & $\begin{array}{l}\text { 1 2 times/day, } \\
45 \sim 60 \text { mins/ } \\
\text { session }\end{array}$ & 260 & SBP; DBP \\
\hline $\begin{array}{l}\mathrm{He}, \\
2015\end{array}$ & $\begin{array}{l}\text { CGMH- } \\
2005\end{array}$ & $\mathrm{EH} 1$ & $\begin{array}{c}28 /-/- \\
42 / 22 / 20 \\
42 / 23 / 19\end{array}$ & $\begin{array}{l}68.51 \pm 2.97 \\
69.24 \pm 2.45\end{array}$ & $\begin{array}{c}\text { AHD } \\
\text { BDJ + AHD } \\
\text { AHD }\end{array}$ & $\begin{array}{l}5 \text { times/week, } \\
30 \text { mins/session }\end{array}$ & 12 & SBP; DBP \\
\hline $\begin{array}{l}\text { Jiang, } \\
2019\end{array}$ & $\begin{array}{l}\text { CGMH- } \\
2010\end{array}$ & $\mathrm{EH} 1,2$ & $50 / 25 / 25$ & $64.67 \pm 3.15$ & $\mathrm{BDJ}+\mathrm{AHD}$ & $\begin{array}{c}\text { At least } 5 \text { days/ } \\
\text { week, } 2 \text { times/ } \\
\text { day, } 30 \text { mins/ } \\
\text { session }\end{array}$ & 12 & SBP; DBP \\
\hline Li, 2015 & $\begin{array}{l}\text { CGMH- } \\
2004\end{array}$ & $\mathrm{EH} 1,2$ & $\begin{array}{c}50 / 26 / 24 \\
30 \\
30\end{array}$ & $\begin{array}{c}65.23 \pm 3.23 \\
\text { NA }\end{array}$ & $\begin{array}{c}\text { AHD } \\
\text { WQX + AHD } \\
\text { AHD }\end{array}$ & $\begin{array}{l}5 \sim 6 \text { times/week, } \\
30 \text { mins/session }\end{array}$ & $\begin{array}{l}12 \\
24\end{array}$ & SBP; DBP \\
\hline $\begin{array}{l}\text { Liang, } \\
2016\end{array}$ & $\begin{array}{l}\text { CGMH- } \\
2010\end{array}$ & NA & $\begin{array}{l}30 / 17 / 13 \\
30 / 16 / 14\end{array}$ & $\begin{array}{l}68.1 \pm 10.1 \\
70.5 \pm 10.2\end{array}$ & $\begin{array}{c}\mathrm{BDJ}+\mathrm{AHD} \\
\mathrm{AHD}\end{array}$ & $\begin{array}{c}2 \text { times/day, } \\
20 \text { mins/session }\end{array}$ & 12 & SBP; DBP \\
\hline $\begin{array}{l}\text { Lin, } \\
2013\end{array}$ & WHO & $\mathrm{EH} 1,2$ & $\begin{array}{l}68 / 31 / 37 \\
59 / 27 / 32\end{array}$ & NA & $\begin{array}{c}\text { WQX + AHD } \\
\text { AHD }\end{array}$ & $\begin{array}{l}6 \text { times/week, } \\
30 \text { mins/session }\end{array}$ & 24 & SBP; DBP \\
\hline $\begin{array}{l}\text { Lin, } \\
2017\end{array}$ & $\begin{array}{c}\text { CGMH- } \\
2010\end{array}$ & $\mathrm{EH} 1$ & $\begin{array}{l}58 /-/- \\
58 /-/-\end{array}$ & $58 \pm 7.48$ & $\begin{array}{c}\mathrm{BDJ}+\mathrm{AHD} \\
\mathrm{AHD}\end{array}$ & $\begin{array}{l}5 \text { days/week, } 1 \\
\text { time/day, } \\
\text { 30 40 mins/ } \\
\text { session }\end{array}$ & 24 & $\begin{array}{l}\text { SBP; DBP; } \\
\text { NO; ET }\end{array}$ \\
\hline $\begin{array}{l}\text { Liu, } \\
2017\end{array}$ & NA & $\mathrm{EH} 1,2$ & $80 / 47 / 33$ & $43 \pm 6.57$ & $\mathrm{TC}+\mathrm{AHD}$ & $\begin{array}{c}2 \text { times/day, } \\
\text { more than } \\
40 \text { mins/session }\end{array}$ & 24 & SBP; DBP \\
\hline $\begin{array}{l}\text { Liu, } \\
2018\end{array}$ & $\begin{array}{c}\text { CGMH- } \\
2010\end{array}$ & $\mathrm{EH} 1,2$ & $77 / 48 / 29$ & $62.4 \pm 2.4$ & $\mathrm{TC}+\mathrm{AHD}$ & $\begin{array}{l}1 \text { time/day, } \\
40 \sim 60 \text { mins/ } \\
\text { session }\end{array}$ & 24 & SBP; DBP \\
\hline $\begin{array}{l}\text { Liu, } \\
2016\end{array}$ & $\begin{array}{c}\text { CGMH- } \\
2015\end{array}$ & EH1, 2 & $\begin{array}{l}35 / 19 / 16 \\
30 / 16 / 14\end{array}$ & $\begin{array}{c}63.1 \pm 2.1 \\
56.33 \pm 7.16\end{array}$ & $\begin{array}{c}\text { AHD } \\
\text { TC + AHD }\end{array}$ & $\begin{array}{l}5 \text { times/week, } \\
40 \text { mins/session }\end{array}$ & 12 & NO; ET \\
\hline
\end{tabular}


TABLE 2: Continued.

\begin{tabular}{|c|c|c|c|c|c|c|c|c|}
\hline Author & $\begin{array}{l}\text { Diagnosis } \\
\text { criteria }\end{array}$ & level of blood pressure & $\begin{array}{l}\text { Sample } \\
\text { N/M/F }\end{array}$ & $\begin{array}{l}\text { Mean age } \\
\text { (years) }\end{array}$ & Interventions & $\begin{array}{c}\text { Exercise } \\
\text { prescription }\end{array}$ & $\begin{array}{l}\text { Length of } \\
\text { intervention } \\
\text { (weeks) }\end{array}$ & $\begin{array}{l}\text { Outcome } \\
\text { measures }\end{array}$ \\
\hline \multirow{3}{*}{$\begin{array}{l}\text { Luo, } \\
2006\end{array}$} & \multirow{3}{*}{$\begin{array}{c}1999 \\
\text { WHO-ISH }\end{array}$} & \multirow{3}{*}{ NA } & $30 / 19 / 11$ & $56.80 \pm 6.78$ & AHD & \multirow{3}{*}{$\begin{array}{c}1 \text { time } / \text { day, } \\
45 \mathrm{mins} / \mathrm{session}\end{array}$} & & \multirow{3}{*}{ SBP; DBP } \\
\hline & & & $44 / 24 / 20$ & $44.74 \pm 12.10$ & $\mathrm{TC}+\mathrm{AHD}$ & & 24 & \\
\hline & & & $40 / 21 / 19$ & $44.86 \pm 13.05$ & AHD & & 24 & \\
\hline $\begin{array}{l}\text { Mao, } \\
2006\end{array}$ & $\begin{array}{c}1999 \\
\text { WHO-ISH }\end{array}$ & NA & $51 / 13 / 38$ & $45 \sim 70$ & $\mathrm{TC}+\mathrm{AHD}$ & $\begin{array}{l}6 \text { times/week, } \\
60 \text { mins/session }\end{array}$ & 8 & $\begin{array}{l}\text { SBP; DBP; } \\
\text { NO; ET }\end{array}$ \\
\hline $\begin{array}{l}\text { Pan, } \\
2010\end{array}$ & $\begin{array}{c}1999 \\
\text { WHO-ISH }\end{array}$ & EH1 & $24 / 14 / 10$ & $62.1 \pm 5.8$ & $\mathrm{BDJ}+\mathrm{AHD}$ & $\begin{array}{l}5 \text { days/week, } 2 \\
\text { times/day, } \\
45 \text { mins/session }\end{array}$ & 24 & SBP; DBP \\
\hline $\begin{array}{l}\text { Shen, } \\
2016\end{array}$ & $\begin{array}{l}\text { CGMH- } \\
2013\end{array}$ & $\mathrm{EH} 1,2$ & $\begin{array}{l}36 /-1- \\
33 /-1-\end{array}$ & $\begin{array}{c}61.4 \pm 7.1 \\
\text { NA }\end{array}$ & $\begin{array}{c}\text { AHD } \\
\text { WQX + AHD } \\
\text { AHD }\end{array}$ & $\begin{array}{l}6 \text { times/week, } \\
60 \text { mins/session }\end{array}$ & 24 & SBP; DBP \\
\hline \multirow[t]{2}{*}{$\begin{array}{l}\text { Sun, } \\
2010\end{array}$} & \multirow[t]{2}{*}{$\begin{array}{l}\text { CGMH- } \\
2005\end{array}$} & \multirow[t]{2}{*}{$\mathrm{EH} 1,2$} & $\begin{array}{c}1932 / 7 / \\
25\end{array}$ & $57.19 \pm 8.09$ & $\mathrm{TC}+\mathrm{AHD}$ & \multirow[t]{2}{*}{$\begin{array}{l}6 \text { times/week, } \\
90 \text { mins/session }\end{array}$} & \multirow[t]{2}{*}{12} & \multirow[t]{2}{*}{ SBP; DBP } \\
\hline & & & $\begin{array}{c}1932 / 10 / \\
22\end{array}$ & $57.25 \pm 5.63$ & AHD & & & \\
\hline $\begin{array}{l}\text { Tang, } \\
2009\end{array}$ & $\begin{array}{c}1999 \\
\text { WHO-ISH }\end{array}$ & $\mathrm{EH} 1,2$ & $\begin{array}{c}2016 / 10 / \\
6\end{array}$ & $63.65 \pm 8.71$ & $\mathrm{TC}+\mathrm{AHD}$ & $\begin{array}{c}3 \sim 5 \text { times/week } \\
\text { 30 60 mins/ } \\
\text { session }\end{array}$ & 24 & SBP; DBP \\
\hline $\begin{array}{l}\text { Wang, } \\
2011\end{array}$ & $\begin{array}{l}\text { CGMH- } \\
2005\end{array}$ & $\mathrm{EH} 1,2$ & $\begin{array}{l}30 /-1- \\
30 /-1-\end{array}$ & $\begin{array}{c}62.79 \pm 7.43 \\
\text { NA }\end{array}$ & $\begin{array}{c}\text { AHD } \\
\text { TC+ AHD } \\
\text { AHD }\end{array}$ & $\begin{array}{l}5 \text { times/week, } \\
60 \mathrm{mins} / \mathrm{session}\end{array}$ & 16 & SBP; DBP \\
\hline Xu, 2016 & $\begin{array}{c}1999 \\
\text { WHO-ISH }\end{array}$ & $\mathrm{EH} 1,2$ & $30 / 17 / 13$ & $38.07 \pm 8.09$ & $\mathrm{TC}+\mathrm{AHD}$ & $\begin{array}{c}2 \text { times/day, } \\
10 \text { mins/session }\end{array}$ & 8 & SBP; DBP \\
\hline $\mathrm{Xu}, 2016$ & $\begin{array}{c}1999 \\
\text { WHO-ISH }\end{array}$ & $\mathrm{EH} 1$ & $50 / 25 / 25$ & $\begin{array}{l}37.63 \pm 9.09 \\
69.38 \pm 7.41\end{array}$ & $\begin{array}{c}\text { AHD } \\
\text { TC + AHD }\end{array}$ & NA & 8 & SBP; DBP \\
\hline $\begin{array}{l}\text { Yang, } \\
2014\end{array}$ & $\begin{array}{l}\text { CGMH- } \\
2010\end{array}$ & $\begin{array}{c}140 \mathrm{mmHg} \leq \mathrm{SBP} \\
<170 \mathrm{mmHg} \text {, or } \\
90 \mathrm{mmHg} \leq \mathrm{DBP}\end{array}$ & $33 / 13 / 20$ & $69.54 \pm 7.37$ & $\mathrm{BDJ}+\mathrm{AHD}$ & $\begin{array}{l}5 \text { times/week, } \\
40 \text { mins/session }\end{array}$ & 24 & SBP; DBP \\
\hline $\begin{array}{l}\text { Zheng, } \\
2014\end{array}$ & $\begin{array}{l}\text { CGMH- } \\
2005\end{array}$ & $<110 \mathrm{mmHg}$ & $34 / 16 / 18$ & $69.23 \pm 3.72$ & $\mathrm{BDJ}+\mathrm{AHD}$ & $\begin{array}{l}5 \text { days/week, } 1 \\
\text { time/day } \\
30 \text { mins/session }\end{array}$ & 12 & $\begin{array}{l}\text { SBP; DBP; } \\
\text { NO; ET }\end{array}$ \\
\hline $\begin{array}{l}\text { Zheng, } \\
2015\end{array}$ & $\begin{array}{l}\text { CGMH- } \\
2010\end{array}$ & NA & $49 / 20 / 29$ & $54.71 \pm 5.43$ & $\mathrm{TC}+\mathrm{AHD}$ & $\begin{array}{l}4 \sim 8 \text { times/week } \\
40 \sim 60 \text { mins/ } \\
\text { session }\end{array}$ & 12 & SBP; DBP \\
\hline $\begin{array}{l}\text { Zhong, } \\
2019\end{array}$ & $\begin{array}{c}\text { CGMH- } \\
2010\end{array}$ & $\mathrm{EH} 1,2$ & $\begin{array}{l}49 / 20 / 29 \\
2010 / 2 / 8\end{array}$ & $\begin{array}{c}55.77 \pm 6.24 \\
66.8 \pm 3.26\end{array}$ & $\begin{array}{c}\text { AHD } \\
\text { TC + AHD }\end{array}$ & $\begin{array}{l}3 \text { times/week, } \\
60 \mathrm{mins} / \text { session }\end{array}$ & 12 & SBP; DBP \\
\hline & & & $2009 / 4 / 5$ & $66.22 \pm 3.27$ & AHD & & & \\
\hline
\end{tabular}

SBP: systolic blood pressure; DBP: diastolic blood pressure; NO: nitric oxide; ET: endothelin; AHD: antihypertensive drugs; TC + AHD: Tai Chi plus antihypertensive drugs; BDJ + AHD: Baduanjin plus antihypertensive drugs; WQX + AHD: Wuqinxi plus antihypertensive drugs; EH: essential hypertension; CGMH: Chinese Guidelines for the Management of Hypertension; and NA: not available.

$(37.4 \%)>\operatorname{AHD}(2.9 \%)$. The quality of the evidence for the ranks of the treatment was low (see Table 5).

3.5.4. DBP Outcome. DBP was assessed in 29 trials $(2100$ participants). The network evidence plot for DBP is shown in Figure 5. In DBP reduction, Tai Chi plus AHD (WMD $-7.56 \mathrm{mmHg}$, 95\% CI: -10.15 to -4.96 ) and Baduanjin plus
AHD (WMD $-4.51 \mathrm{mmHg}$, 95\% CI: -7.38 to -1.65 ) were superior to AHD alone; there was no statistically significant difference in other results (see Figure 6 and Table 4). According to SUCRA, the interventions to reduce DBP are ranked in probability (see Table 4 and Figure 7): TC + AHD $(95.4 \%)>\mathrm{BDJ}+\mathrm{AHD} \quad(57.6 \%)>\mathrm{WQX}+\mathrm{AHD} \quad(42.1 \%)$ $>$ AHD (4.9\%). The quality of the evidence for the ranks of the treatment was low (see Table 5). 


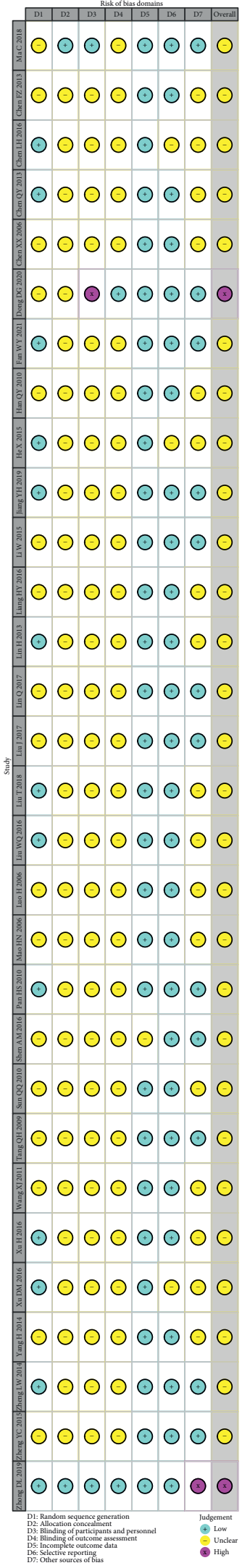

Figure 2: Traffic light plot of ROB.
3.5.5. NO Outcome. NO was assessed in 6 RCTs (388 participants). The network evidence plot for $\mathrm{NO}$ is shown in Figure 5. In increasing NO, Baduanjin plus AHD (WMD $4.26 \mu \mathrm{mol} / \mathrm{L}, 95 \% \mathrm{CI}: 2.68$ to 5.83 ) was superior to AHD alone; there was no statistically significant difference in other results (see Figure 6 and Table 4). According to SUCRA, the interventions to increase NO are ranked in probability (see Table 4 and Figure 7): BDJ + AHD (94.7\%) $>$ TC + AHD $(51.7 \%)>$ AHD $(3.6 \%)$. The quality of the evidence for the ranks of the treatment was low (see Table 5).

3.5.6. ET Outcome. ET was assessed in 6 RCTs (390 participants). The network evidence plot for ET is shown in Figure 5. In ET reduction, Tai Chi plus AHD (WMD -7.64 pg/ml, 95\% CI: -10.46 to -4.83 ) and Baduanjin plus AHD (WMD $-9.23 \mathrm{pg} / \mathrm{ml}, 95 \% \mathrm{CI}:-10.85$ to -7.61 ) were statistically superior to AHD alone; there was no statistically significant difference in other results (see Figure 6 and Table 4). According to SUCRA, the interventions to decrease ET are ranked in probability (see Table 4 and Figure 7): $\mathrm{BDJ}+\mathrm{AHD}(91.4 \%)>\mathrm{TC}+\mathrm{AHD}(58.6 \%)>\mathrm{AHD}(0 \%)$. The quality of the evidence for the ranks of the treatment was low (see Table 5).

\section{Discussion}

4.1. Summary of Findings. The results indicated that combination therapies including Tai Chi plus AHD and Baduanjin plus AHD were superior to AHD alone, and Tai Chi was more effective than other traditional exercises at lowering SBP. Compared with AHD alone, Tai Chi plus AHD and Baduanjin Plus AHD produced a statistically significant DBP reduction. Compared with AHD alone, Baduanjin plus AHD produced a statistically significant $\mathrm{NO}$ increment, while that of Tai Chi plus AHD was not statistically significant. In terms of ET, both Baduanjin plus AHD and Tai Chi plus AHD produced a statistically significant reduction compared with $\mathrm{AHD}$ alone, while the difference between the two exercise types was not statistically significant.

Due to the lack of direct comparisons, there was no closed loop between different interventions, so node splitting method was not used to detect inconsistency; besides, the overall methodological quality of included studies was low, which increased the uncertainty of the results and required careful interpretation of the results.

4.2. Clinical Implications. According to previous research results, traditional Chinese fitness exercises (Tai Chi, Baduanjin, and Wuqinxi) as adjuvant therapy are relatively easy to learn and less intense, especially suitable for longterm training of elderly patients with $\mathrm{EH}$, which can enhance the antihypertensive effect, reduce the dosage of antihypertensive drugs, and ease the economic burden $[43,57,60,62-65]$. However, compared with 
Random sequence generation

Allocation concealment

Blinding of participants and personnel

Blinding of outcome assessment

Incomplete outcome data

Selective reporting

Other sources of bias
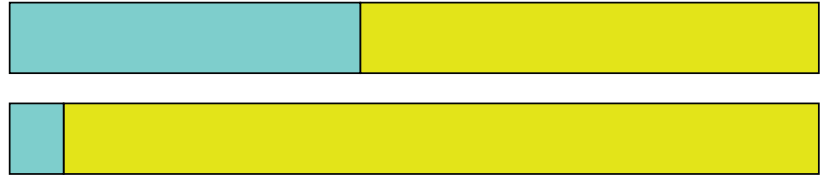

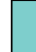
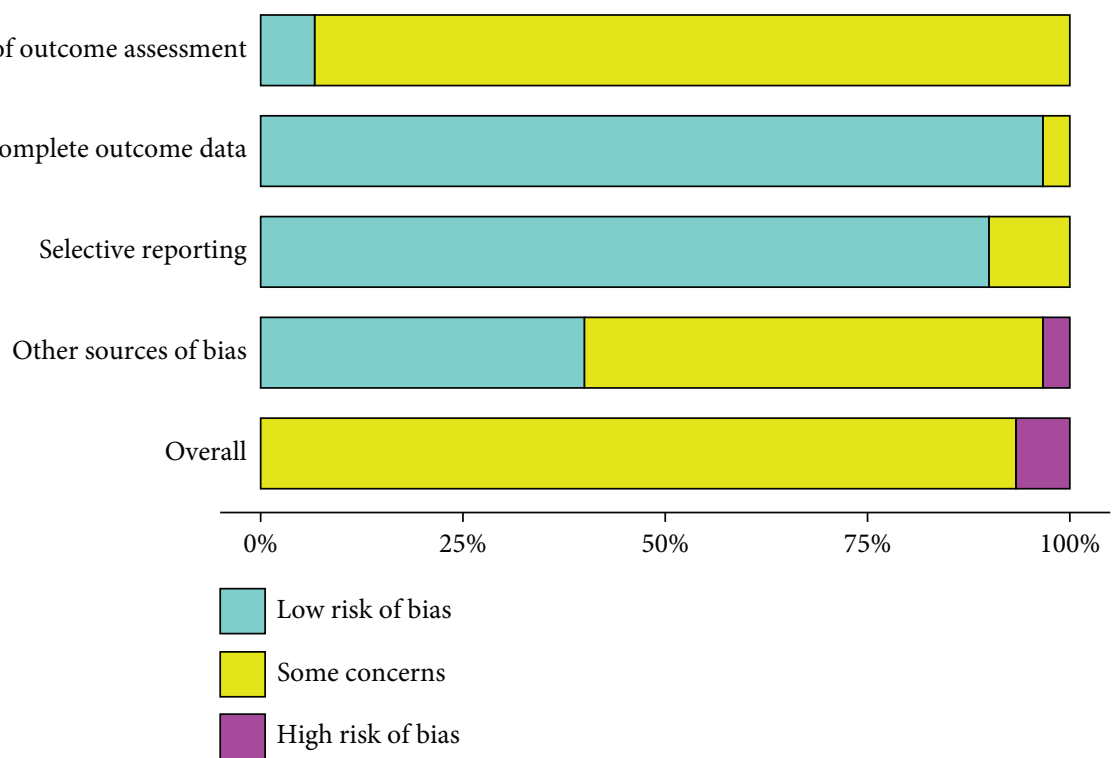

FIgURE 3: Summary plot of ROB.

TABLE 3: Results of the pairwise meta-analysis.

\begin{tabular}{|c|c|c|c|c|c|c|c|c|}
\hline Comparison & $n$ & $\begin{array}{c}\text { SBP } \\
\text { MD (95\% CI) }\end{array}$ & $n$ & $\begin{array}{c}\text { DBP } \\
\text { MD (95\% CI) }\end{array}$ & $n$ & $\begin{array}{c}\text { NO } \\
\text { MD (95\% CI) }\end{array}$ & $n$ & $\begin{array}{c}\text { ET } \\
\text { MD }(95 \% \text { CI })\end{array}$ \\
\hline TC + AHD vs AHD & 15 & $-12.25(-16.22$ to -8.28$)$ & 15 & $-7.58(-9.98$ to -5.19$)$ & 3 & $3.05(-1.32$ to 7.43$)$ & 2 & $-11.94(-25.29$ to 1.41$)$ \\
\hline $\mathrm{BDJ}+\mathrm{AHD}$ vs $\mathrm{AHD}$ & 11 & $-6.92(-8.70$ to -5.14$)$ & 11 & $-4.50(-7.08$ to -1.92$)$ & 3 & $4.42(3.26$ to 5.58$)$ & 4 & $-9.23(-10.85$ to -7.61$)$ \\
\hline WQX + AHD vs AHD & 3 & $-4.10(-9.39$ to 1.19$)$ & 3 & $-3.23(-9.98$ to 3.52$)$ & - & & - & \\
\hline
\end{tabular}

SBP: systolic blood pressure; DBP: diastolic blood pressure; NO: nitric oxide; ET: endothelin; AHD: antihypertensive drugs; TC + AHD: Tai Chi plus antihypertensive drugs; BDJ + AHD: Baduanjin plus antihypertensive drugs; WQX + AHD: Wuqinxi plus antihypertensive drugs; MD: mean difference; and CI: credible intervals. The bold values mean that the results are statistically significant.

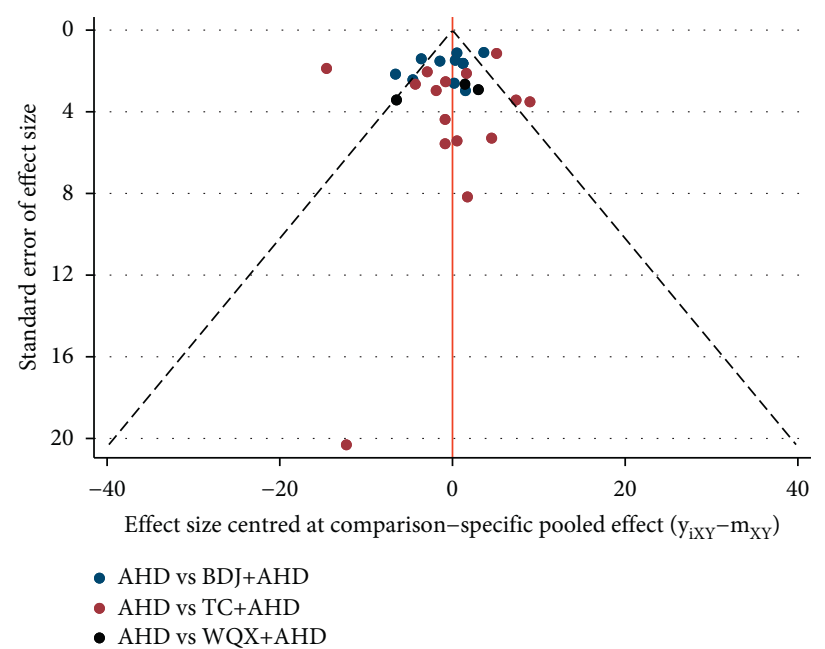

(a)

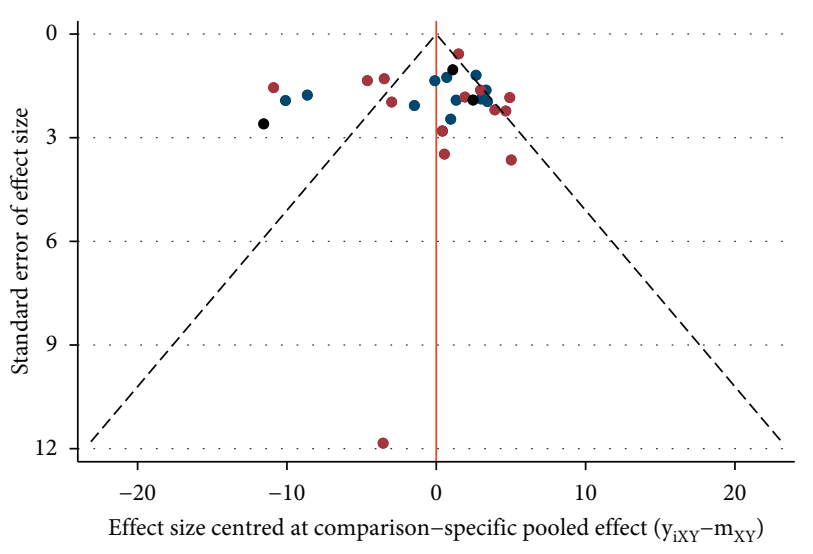

- AHD vs BDJ+AHD

- AHD vs TC+AHD

- AHD vs WQX+AHD

FIGURE 4: Continued. 


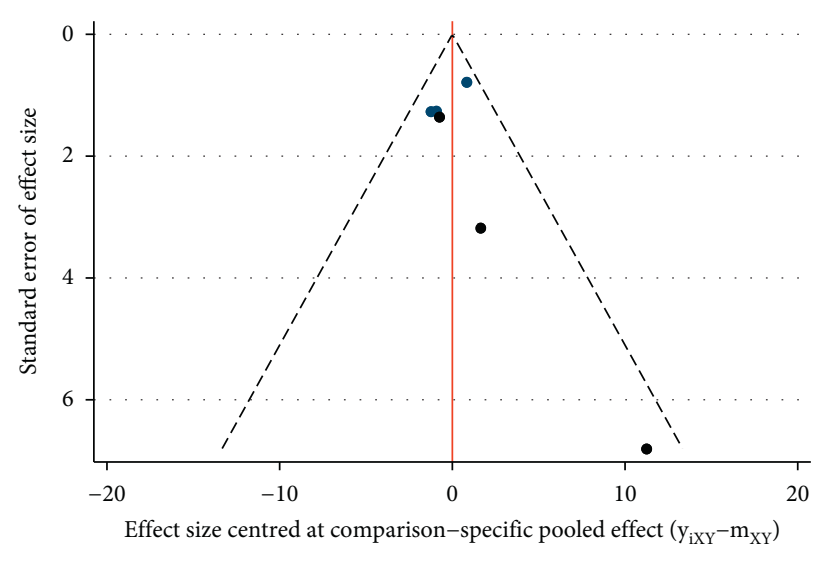

- AHD vs BDJ+AHD

- $\mathrm{AHD}$ vs TC+AHD

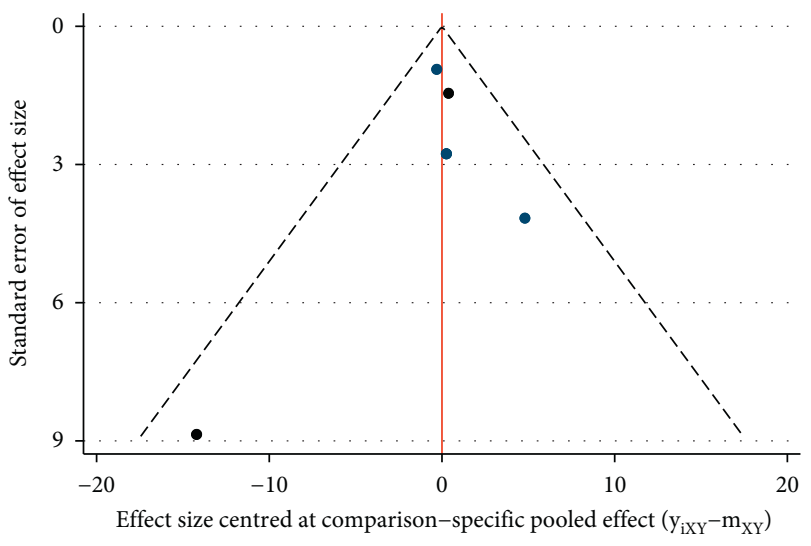

- AHD vs BDJ+AHD

- AHD vs TC+AHD

(c)

(d)

FIGURE 4: Comparison-adjusted funnel plots. The points of different colors represent a direct comparison of two interventions: (a) SBP; (b) DBP; (c) NO; and (d) ET.

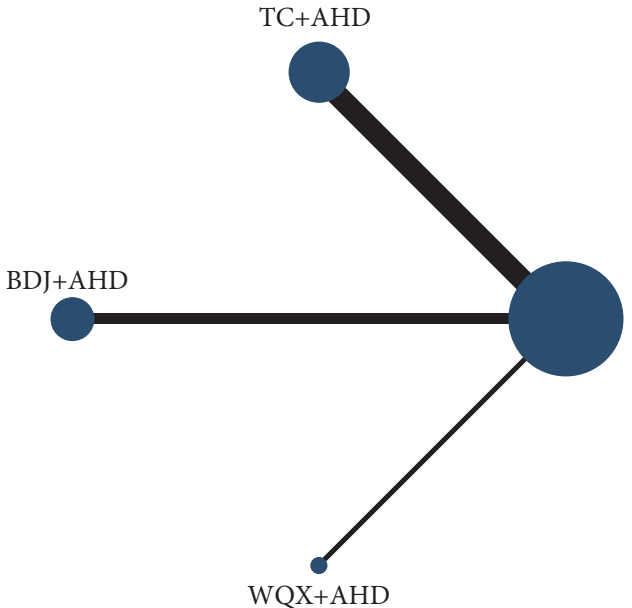

(A)

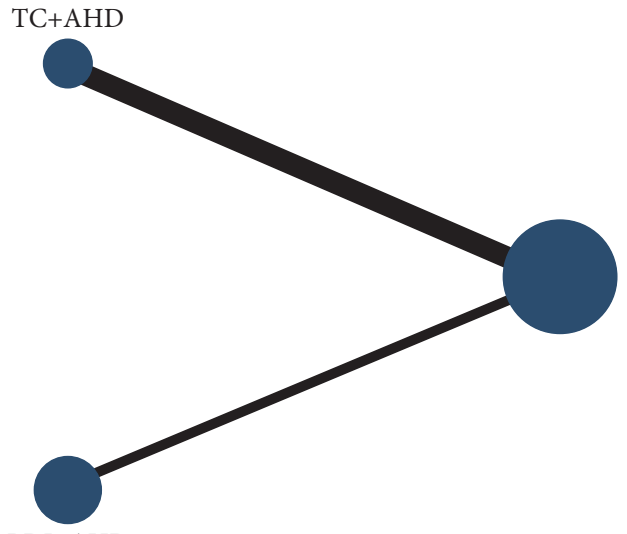

$\mathrm{BDJ}+\mathrm{AHD}$

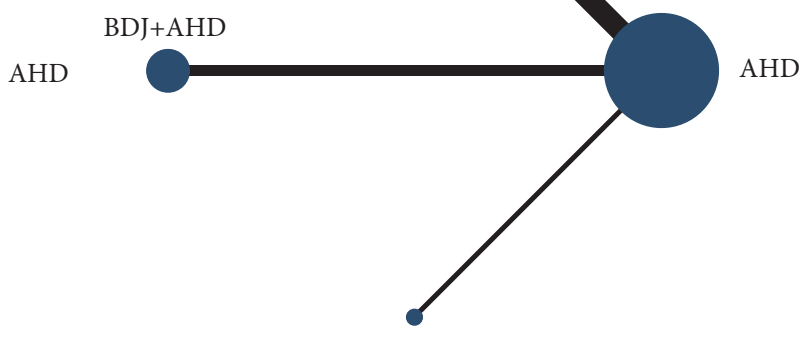

WQX+AHD

(B)

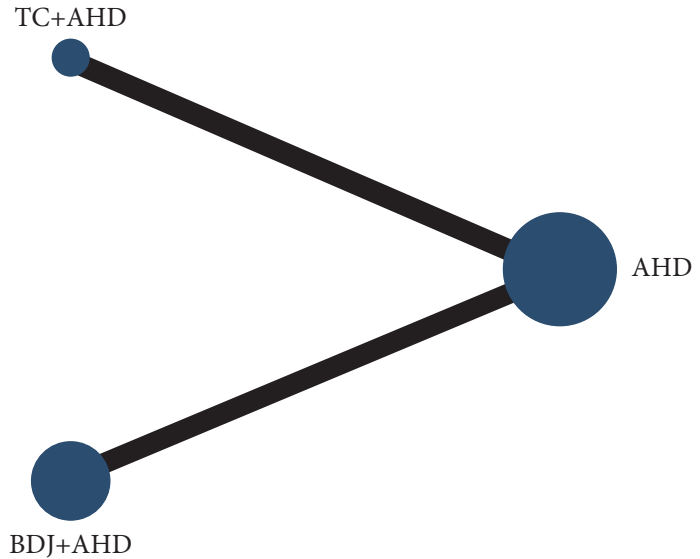

(D)

FIGURE 5: Network of direct comparisons formed by included studies. The node size and line thickness are proportionate to the number of studies: (A) SBP; (B) DBP; (C) NO; (D) ET. 


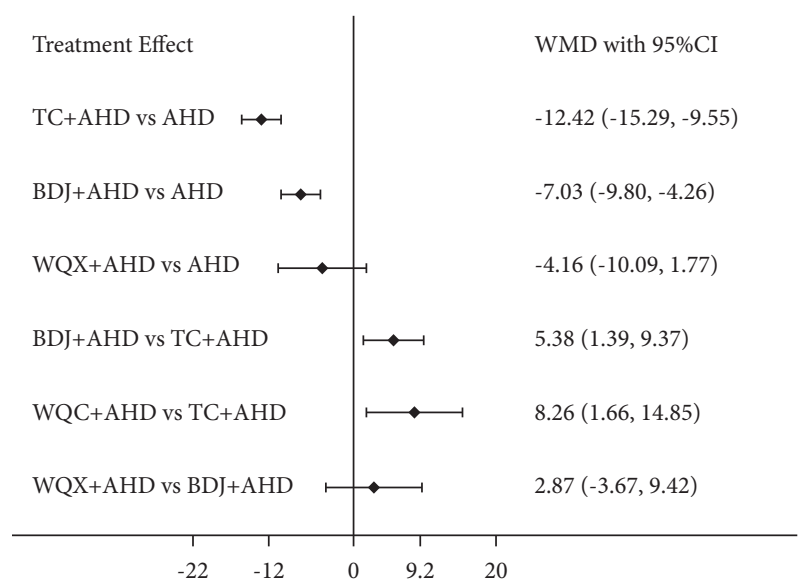

(A)

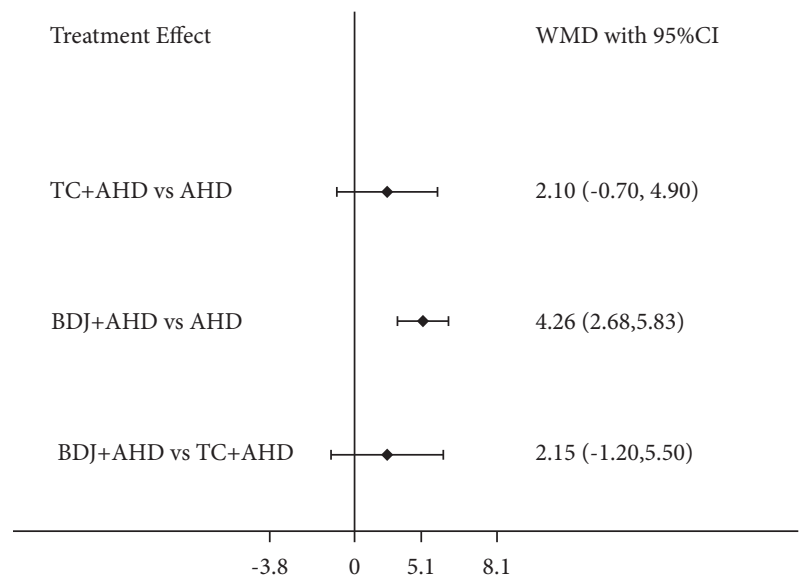

(C)

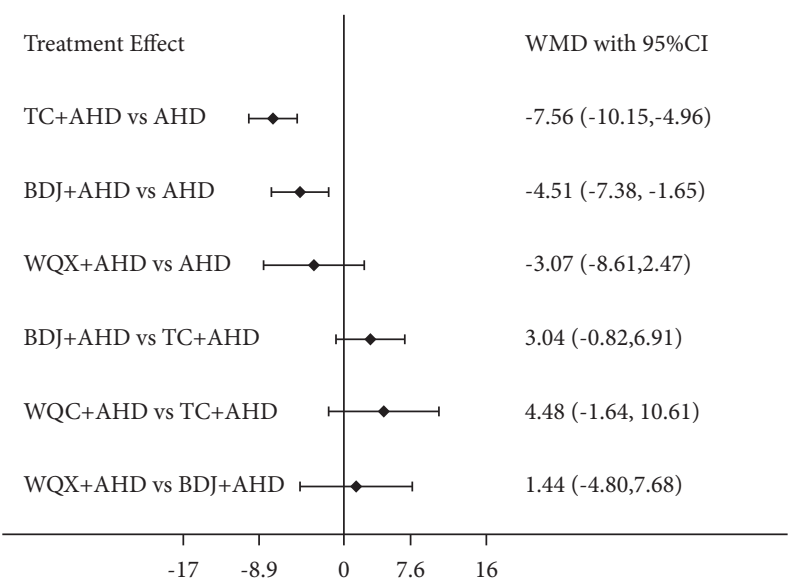

(B)

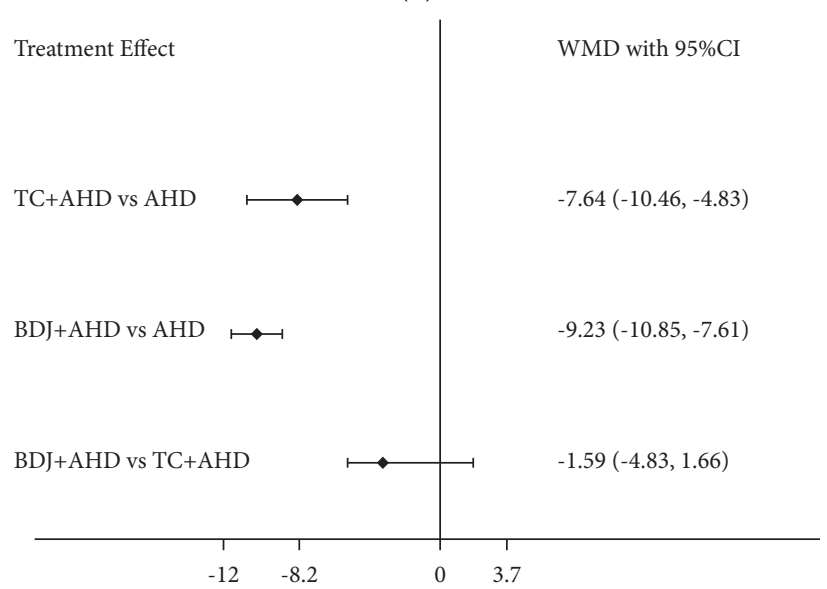

(D)

Figure 6: Effect size of different comparators presented in weighted mean difference (95\% CI): (A) SBP; (B) DBP; (C) NO; (D) ET.

TABLE 4: Results of network meta-analysis of SBP, DBP, NO, and ET.

\begin{tabular}{|c|c|c|c|c|c|}
\hline \multirow{2}{*}{ Treatment type } & \multirow{2}{*}{$\begin{array}{c}\text { No adjustment } \\
\text { Pooled WMD }(95 \% \mathrm{Cl})\end{array}$} & \multicolumn{3}{|c|}{ Likelihood (\%) of being } & \multirow{2}{*}{ SUCRA (\%) } \\
\hline & & $P$ value & Best & Worst & \\
\hline \multicolumn{6}{|l|}{ SBP } \\
\hline AHD & Reference & - & 0 & 91.5 & 2.9 \\
\hline $\mathrm{TC}+\mathrm{AHD}$ & $-12.42(-15.29$ to -9.55$)$ & $<0.001$ & 98.5 & 0 & 99.5 \\
\hline $\mathrm{BDJ}+\mathrm{AHD}$ & $-7.03(-9.80$ to -4.26$)$ & $<0.001$ & 0.5 & 0 & 60.2 \\
\hline WQX + AHD & $-4.16(-10.09$ to 1.77$)$ & 0.169 & 1 & 8.6 & 37.4 \\
\hline \multicolumn{6}{|l|}{ DBP } \\
\hline AHD & Reference & - & 0 & 85.4 & 4.9 \\
\hline $\mathrm{TC}+\mathrm{AHD}$ & $-7.56(-10.15$ to -4.96$)$ & $<0.001$ & 87.2 & 0 & 95.4 \\
\hline $\mathrm{BDJ}+\mathrm{AHD}$ & $-4.51(-7.38$ to -1.65$)$ & 0.002 & 5.3 & 0.1 & 57.6 \\
\hline WQX + AHD & $-3.07(-8.61$ to 2.47$)$ & 0.277 & 7.5 & 14.5 & 42.1 \\
\hline \multicolumn{6}{|l|}{ NO } \\
\hline $\mathrm{AHD}$ & Reference & - & 0 & 92.8 & 3.6 \\
\hline $\mathrm{TC}+\mathrm{AHD}$ & $2.10(-0.70$ to 4.90$)$ & 0.141 & 10.5 & 7.2 & 51.7 \\
\hline $\mathrm{BDJ}+\mathrm{AHD}$ & $4.26(2.68$ to 5.83$)$ & $<0.001$ & 89.5 & 0 & 94.7 \\
\hline \multicolumn{6}{|l|}{ ET } \\
\hline AHD & Reference & - & 0 & 100 & 0 \\
\hline $\mathrm{TC}+\mathrm{AHD}$ & $-7.64(-10.46$ to -4.83$)$ & $<0.001$ & 17.2 & 0 & 58.6 \\
\hline $\mathrm{BDJ}+\mathrm{AHD}$ & $-9.23(-10.85$ to -7.61$)$ & $<0.001$ & 82.8 & 0 & 91.4 \\
\hline
\end{tabular}

SBP: systolic blood pressure; DBP: diastolic blood pressure; NO: nitric oxide; ET: endothelin; AHD: antihypertensive drugs; TC + AHD: Tai Chi plus antihypertensive drugs; BDJ + AHD: Baduanjin plus antihypertensive drugs; WQX + AHD: Wuqinxi plus antihypertensive drugs; WMD: weighted mean difference; CI: credible intervals; and SUCRA: surface under the cumulative ranking curve. The bold values mean that the results are statistically significant. 


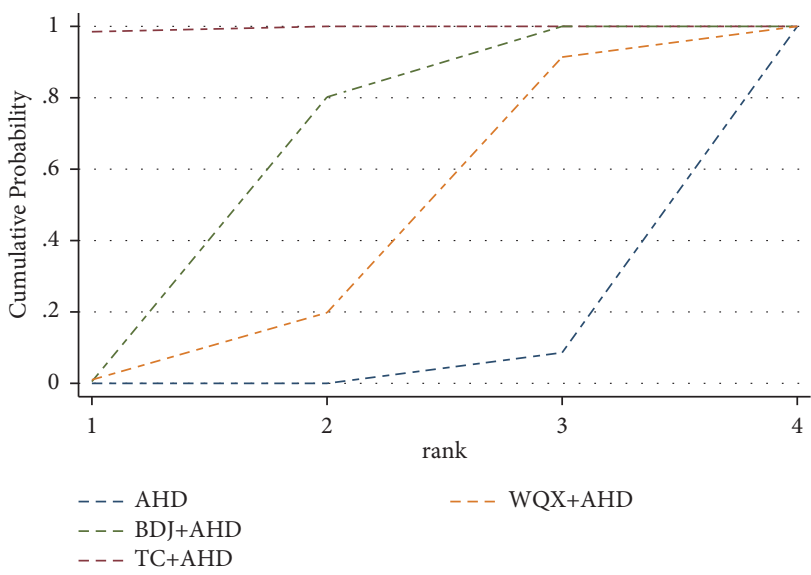

(a)

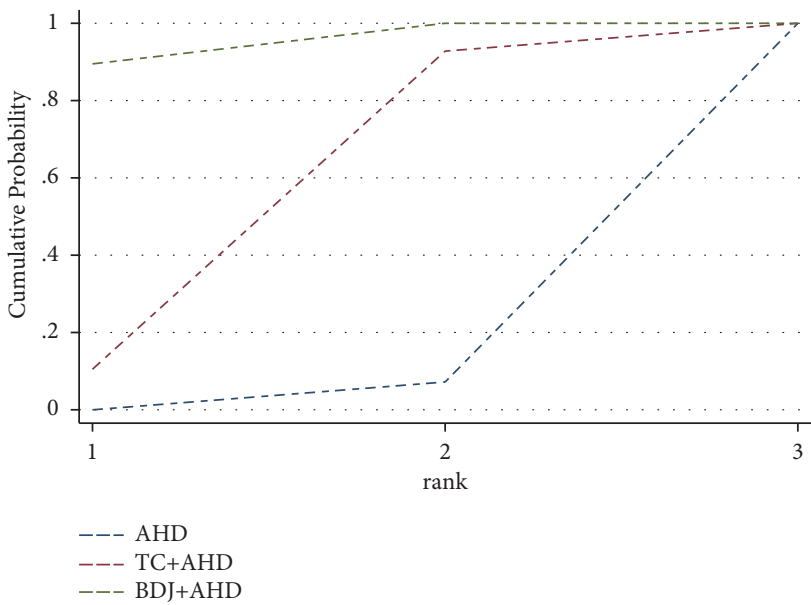

(c)

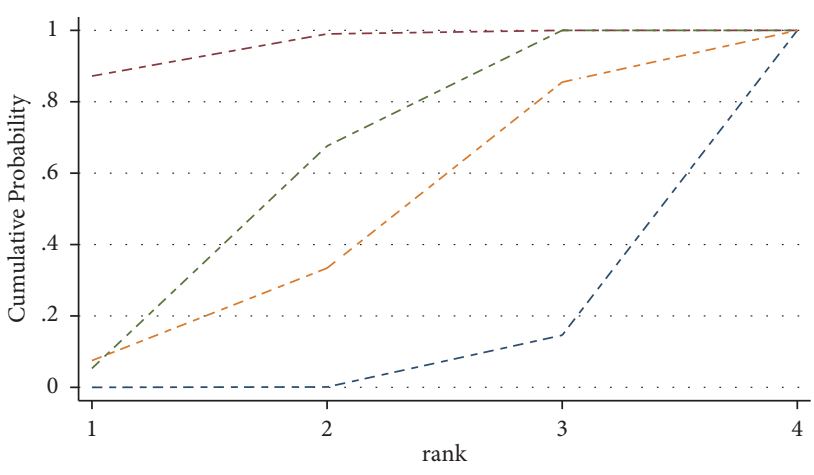

$$
\begin{aligned}
& -- \text { AHD } \\
& --- \text { BDJ+AHD } \\
& --- \text { TC+AHD }
\end{aligned}
$$

(b)

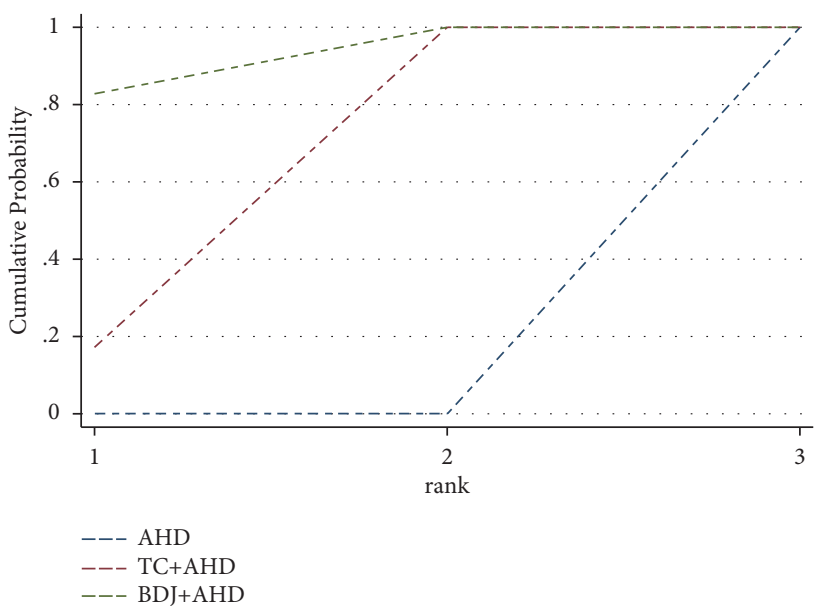

(d)

Figure 7: Cumulative ranking plots. Ranking of interventions are proportionate to the size of surface under the dotted line: (a) SBP; (b) DBP; (c) NO; (d) ET.

TABLE 5: Summary of confidence in ranking of interventions for outcomes (GRADE approach) in studies.

\begin{tabular}{lcc}
\hline Outcome & Confidence & \multicolumn{1}{c}{ Downgrading due to } \\
\hline SBP & Low & Study limitations; indirectness \\
DBP & Low & Study limitations; indirectness \\
NO & Low & Study limitations; indirectness \\
ET & Low & Study limitations; indirectness \\
\hline
\end{tabular}

SBP: systolic blood pressure; DBP: diastolic blood pressure; NO: nitric oxide; and ET: endothelin.

antihypertensive drugs, the effects of traditional Chinese fitness exercises on $\mathrm{EH}$ remain understudied and evidence quality hierarchy of most of literatures in this field is at lower levels, meaning that it is necessary to carry out further highquality randomized controlled trials on traditional Chinese fitness exercise-assisted treatment of $\mathrm{EH}$ in the future.

Based on the NMA results in this study, as adjunctive kinesiotherapy for $\mathrm{EH}$, Tai Chi and Baduanjin have a statistically significant benefit in reducing the levels of SBP, DBP, and ET. Baduanjin has a statistically significant benefit in increasing the level of NO, while Wuqinxi may be the least effective. These findings of this NMA may be useful for clinicians and health professionals to select appropriate traditional Chinese fitness exercise as an adjuntive kinesiotherapy or early intervention prescription for patients with $\mathrm{EH}$.

4.3. Strength and Limitations. There has been an increasing number of studies on traditional Chinese fitness exercises as adjuvant therapies for $\mathrm{EH}$ in recent years. However, our previous study rarely directly compared the effectiveness of different traditional Chinese fitness exercises. Traditional meta-analysis cannot compare multiple interventions, but NMA can address this issue. NMA attempts to integrate decision-making evidences through evaluating relative effectiveness of two or more alternative interventions in the same situation [66]. Consequently, in this study, we not only compared the effectiveness of AHD alone with traditional Chinese fitness exercises combined with AHD, but also calculated cumulative rankings for identifying superiority among the three traditional Chinese fitness exercises using Stata program based on the frequentist framework. This work could provide guidance and information for clinicians 
to choose traditional Chinese exercises as an adjunct therapy for patients with $\mathrm{EH}$.

However, several limitations in this NMA should be noted. First, among the included literatures, there were only three literatures about Wuqinxi as an adjuvant therapy for $\mathrm{EH}$, which was less than Tai Chi and Baduanjin. The estimates for the efficacy of Wuqinxi as adjuvant therapy for $\mathrm{EH}$ were open to considerable uncertainty as the number of studies was small, which leads to the current evidence and potential findings that still require careful interpretation. Second, since no RCT compared directly among the three traditional Chinese fitness exercises currently, we could only use indirect evidence to compare the efficacy of three traditional Chinese exercises. If the distribution of effect modifiers between different direct comparisons is unbalanced, the associated indirect comparisons will be biased [67]. Third, due to insufficient sample size of some exercise trials, confounding factors cannot be adequately controlled, leading to relatively unreliable estimates of therapeutic effects. Fourth, the quality of evidence in this NMA was low according to GRADE criteria. Therefore, if high-quality evidence is obtained, the effect ranking order of interventions and pooled effect sizes may change. Fifth, although the current review is not registered, which may lead to potential bias, we still strictly follow the steps of the system review.

\section{Conclusions}

Based on the current results, we can reach the following conclusion. Compared with AHD alone, both Tai Chi plus AHD and Baduanjin plus AHD show significant benefit in regulating SBP, DBP, and ET. Among three traditional Chinese fitness exercises, Tai Chi may be the best as an adjunctive therapy for SBP reduction. These findings provide evidence for the therapeutic benefit of either Tai Chi or Baduanjin exercise as an adjunct therapy for patients with $\mathrm{EH}$. The overall methodological quality of included studies was low, so current results need to be interpreted with caution, and it is necessary to carry out further high-quality RCTs on traditional Chinese fitness exercise-assisted treatment of $\mathrm{EH}$ in the future.

\section{Data Availability}

The datasets used during the current study are available from the corresponding author upon reasonable request.

\section{Conflicts of Interest}

The authors declare that they have no conflicts of interest regarding the publication of this paper.

\section{Authors' Contributions}

Lulu Dai and Yuerong Jiang put forward the idea of this study and designed the method of this systematic review. Lulu Dai, Yuerong Jiang, and Peili Wang searched the articles, conducted data collection and extraction, and analyzed the data. Keji Chen provided guidelines for this systematic review and network meta-analysis. The authors approved the final version of the manuscript for publication.

\section{Acknowledgments}

This research was financially supported by National Natural Science Foundation of China (81373822).

\section{Supplementary Materials}

Supplementary Appendix 1. Search strategies of CENTRAL, PubMed, EMBASE, and Web of Science. Supplementary Appendix 2. Forest plots of pairwise meta-analysis of effect of traditional Chinese exercise on essential hypertension. (Supplementary Materials)

\section{References}

[1] Y. Kokubo and C. Matsumoto, "Hypertension is a risk factor for several types of heart disease: review of prospective studies," Advances in Experimental Medicine \& Biology, vol. 956, pp. 419-426, 2017.

[2] H. J. L. Heerspink, T. Ninomiya, S. Zoungas et al., "Effect of lowering blood pressure on cardiovascular events and mortality in patients on dialysis: a systematic review and metaanalysis of randomised controlled trials," The Lancet, vol. 373, no. 9668, pp. 1009-1015, 2009.

[3] S. S. Lim, T. Vos, A. D. Flaxman et al., "A comparative risk assessment of burden of disease and injury attributable to 67 risk factors and risk factor clusters in 21 regions, 1990-2010: a systematic analysis for the Global Burden of Disease Study 2010," The Lancet, vol. 380, no. 9859, pp. 2224-2260, 2012.

[4] "GBD 2015 Risk Factors Collaborators, "Global, regional, and national comparative risk assessment of 79 behavioural, environmental and occupational, and metabolic risks or clusters of risks, 1990-2015: a systematic analysis for the Global Burden of Disease Study 2015," Lancet (North American Edition), vol. 388, no. 10053, pp. 1659-1724, 2016.

[5] S. Oparil, M. C. Acelajado, G. L. Bakris et al., "Hypertension," Nature reviews. Disease primers, vol. 4, p. 18014, 2018.

[6] E. Reeve, V. Jordan, W. Thompson et al., "Withdrawal of antihypertensive drugs in older people," Cochrane Database of Systematic Reviews, vol. 6, no. 6, Article ID CD012572, 2020.

[7] S. D. Ross, K. S. Akhras, S. Zhang, M. Rozinsky, and L. Nalysnyk, "Discontinuation of antihypertensive drugs due to adverse events: a systematic review and meta-analysis," Pharmacotherapy: The Journal of Human Pharmacology and Drug Therapy, vol. 21, no. 8, pp. 940-953, 2001.

[8] C. Thomopoulos, G. Parati, and A. Zanchetti, "Effects of blood-pressure-lowering treatment in hypertension," Journal of Hypertension, vol. 34, no. 10, pp. 1921-1932, 2016.

[9] B. Williams, G. Mancia, W. Spiering et al., "2018 practice guidelines for the management of arterial hypertension of the European society of cardiology and the European society of hypertension," Journal of Hypertension, vol. 36, no. 12, pp. 2284-2309, 2018.

[10] F. Manfredini, A. M. Malagoni, S. Mandini et al., "Sport therapy for hypertension: why, how, and how much?" Angiology, vol. 60, no. 2, pp. 207-216, 2009.

[11] H. Thiele, C. Pohlink, and G. Schuler, "[Hypertension and exercise. Sports methods for the hypertensive patient]," Herz, vol. 29, no. 4, pp. 401-405, 2004. 
[12] R. H. Fagard and V. A. Cornelissen, "Effect of exercise on blood pressure control in hypertensive patients," European Journal of Cardiovascular Prevention \& Rehabilitation, vol. 14, no. 1, pp. 12-17, 2007.

[13] G. Saco-Ledo, P. L. Valenzuela, G. Ruiz-Hurtado, L. M. Ruilope, and A. Lucia, "Exercise reduces ambulatory blood pressure in patients with hypertension: a systematic review and meta-analysis of randomized controlled trials," Journal of the American Heart Association, vol. 9, no. 24, Article ID e18487, 2020.

[14] I. C. Moraes-Silva, C. T. Mostarda, A. C. Silva-Filho, and M. C. Irigoyen, "Hypertension and exercise training: evidence from clinical studies," Advances in Experimental Medicine \& Biology, vol. 1000, pp. 65-84, 2017.

[15] P. K. Whelton, R. M. Carey, W. S. Aronow et al., "2017 ACC/ AHA/AAPA/ABC/ACPM/AGS/APhA/ASH/ASPC/NMA/ PCNA Guideline for the prevention, detection, evaluation, and management of high blood pressure in adults: executive summary: a report of the American college of cardiology/ American heart association task force on clinical practice guidelines," Journal of the American College of Cardiology, vol. 71, no. 19, pp. 2199-2269, 2018.

[16] H. Naci, M. Salcher-Konrad, S. Dias et al., "How does exercise treatment compare with antihypertensive medications? A network meta-analysis of 391 randomised controlled trials assessing exercise and medication effects on systolic blood pressure," British Journal of Sports Medicine, vol. 53, no. 14, pp. 859-869, 2019.

[17] B.-y. Shao, X.-t. Zhang, R. W. M. Vernooij et al., "The effectiveness of Baduanjin exercise for hypertension: a systematic review and meta-analysis of randomized controlled trials," BMC Complementary Medicine and Therapies, vol. 20, no. 1, p. 304, 2020.

[18] D. Zhong, J. Li, H. Yang et al., "Tai chi for essential hypertension: a systematic review of randomized controlled trials," Current Hypertension Reports, vol. 22, no. 3, p. 25, 2020.

[19] Y. Guan, Y. Hao, Y. Guan, and H. Wang, "Effects of Tai Chi on essential hypertension and related risk factors: a meta-analysis of randomized controlled trials," Journal of Rehabilitation Medicine, vol. 52, no. 5, Article ID jrm00057, 2020.

[20] Y. Guan, Y. Hao, Y. Guan, and H. Wang, "Effects of Baduanjin exercise on essential hypertension: a meta-analysis of randomized controlled trials," Medicine (Baltimore), vol. 99, no. 32, Article ID e21577, 2020.

[21] X. Xiong, P. Wang, S. Li, Y. Zhang, and X. Li, "Effect of Baduanjin exercise for hypertension: a systematic review and meta-analysis of randomized controlled trials," Maturitas, vol. 80, no. 4, pp. 370-378, 2015.

[22] G. Y. Yeh, C. Wang, P. M. Wayne, and R. S. Phillips, "The effect of tai chi exercise on blood pressure: a systematic review," Preventive Cardiology, vol. 11, no. 2, pp. 82-89, 2008.

[23] M. S. Lee, E.-N. Lee, J.-I. Kim, and E. Ernst, "Tai chi for lowering resting blood pressure in the elderly: a systematic review," Journal of Evaluation in Clinical Practice, vol. 16, no. 4, pp. 818-824, 2010.

[24] X. Dong, M. Ding, and X. Yi, "Meta-analysis of randomized controlled trials of the effects of tai chi on blood pressure," Evidence-based Complementary and Alternative Medicine, vol. 2020, Article ID 8503047, 2020.

[25] L. Hartley, N. Flowers, M. S. Lee, E. Ernst, and K. Rees, "Tai chi for primary prevention of cardiovascular disease," Cochrane Database of Systematic Reviews, vol. 4, Article ID CD010366, 2014.
[26] Y. C. Zheng, L. Chen, and J. Q. Yang, “Taijiquan systematic reviews of primary effects on blood pressure and quality of life of patients with hypertension," Journal of Liaoning University of Traditional Chinese Medicine, vol. 17, no. 4, pp. 143-146, 2015.

[27] J. Ioannidis, "Next-generation systematic reviews: prospective meta-analysis, individual-level data, networks and umbrella reviews," British Journal of Sports Medicine, vol. 51, no. 20, pp. 1456-1458, 2017.

[28] G. Salanti, A. E. Ades, and J. P. A. Ioannidis, "Graphical methods and numerical summaries for presenting results from multiple-treatment meta-analysis: an overview and tutorial," Journal of Clinical Epidemiology, vol. 64, no. 2, pp. 163-171, 2011.

[29] Joint Committee for Guideline Revision, "2018 Chinese guidelines for prevention and treatment of hypertension-A report of the revision committee of Chinese guidelines for prevention and treatment of hypertension," Journal of Geriatric Cardiology, vol. 16, no. 3, pp. 182-241, 2019.

[30] A. V. Chobanian, G. L. Bakris, H. R. Black et al., "The Seventh report of the Joint national committee on prevention, detection, evaluation, and treatment of high blood PressureThe JNC 7 report," Journal of the American Medical Association, vol. 289, no. 19, pp. 2560-2572, 2003.

[31] J. P. T. Higgins and S. Green, Cochrane Handbook for Systematic Reviews of Interventions Version 5.1.0, The Cochrane Collaboration, London, UK, 2011, https://www.handbook. cochrane.org.

[32] G. Salanti, C. Del Giovane, A. Chaimani, D. M. Caldwell, and J. P. T. Higgins, "Evaluating the quality of evidence from a network meta-analysis," PLoS One, vol. 9, no. 7, p. e99682, 2014.

[33] C. Ma, W. Zhou, Q. Tang, and S. Huang, "The impact of group-based Tai chi on health-status outcomes among community-dwelling older adults with hypertension," Heart \& Lung, vol. 47, no. 4, pp. 337-344, 2018.

[34] F. Z. Chen and Q. B. Lv, "Effects of Taijiquan on blood pressure in patients with hypertension," Today Nurse, vol. 4, pp. 18-19, 2013.

[35] L. H. Chen, "Application of Baduanjin in rehabilitation nursing of elderly patients with hypertension," Journal of Frontiers of Medicine, vol. 6, no. 22, pp. 340-341, 2016.

[36] Q. Y. Chen, "The Mechanism of Baduanjin qigong reduce blood pressure of type 1 hypertension-from vascular endothelium function," M. S. thesis, Fujian University of Traditional Chinese Medicine, Fujian, China, 2013.

[37] X. X. Chen and H. Q. Lv, "Effects of Taijiquan exercise on hypertension patients' NO consistency in Plasma, the activity of RBC Na+-K+, ATPase and Ca2+-Mg2+ATPase," Journal of Beijing Sport University, vol. 29, no. 10, pp. 1359-1361, 2006.

[38] D. G. Dong, Z. D. Yu, and Z. S. Yu, "The intervention effect of Baduanjin fitness qigong on patients with phlegm-dampness hypertension," Chinese Journal of Applied Physiology, vol. 36, no. 2, pp. 157-160, 2020.

[39] W. Y. Fan, L. W. Zheng, F. Chen et al., "Influence of Baduanjin on anxiety and depression in 38 elderly patients with essential hypertension," Fujian Journal of Traditional Chinese Medicine, vol. 52, no. 2, pp. 11-13, 2021.

[40] Q. Y. Han, X. F. Huang, L. Li, and L. Q. Chen, "The effect of shadow boxing exercise on the long-term quality of life in middle-aged and elderly patients with primary hypertension," Chinses Journal of Modern Nursin, vol. 16, no. 14, pp. 16171619, 2010. 
[41] X. He, "Rehabilitation therapeutic effect of baduanjin training in aged patients with hypertension," Chinese Journal of Cardiovascular Rehabilitation Medicine, vol. 24, no. 3, pp. 252-254, 2015.

[42] Y. H. Jiang, G. L. Fu, Q. P. Wang, X. P. Bao, and X. X. Liu, "Effect of Baduanjin on elderly patients with liver-yang hypertension and Anxiety," Journal of Qilu Nursing, vol. 25, no. 19, pp. 104-106, 2019.

[43] W. Li, H. Li, and H. R. Luan, "Study for the intervention effect of Wuqinxi for elder hypertension patients with obesity," Medical Information, vol. 28, no. 9, pp. 48-49, 2015.

[44] H. Y. Liang, C. Y. Huang, and D. H. Li, "Effects of Baduanjin on blood pressure and quality of life in patients with isolated systolic hypertension," Chinese Manipulation \& Rehabilitation Medicine, vol. 7, no. 16, pp. 12-15, 2016.

[45] H. Lin and S. J. Huang, "The promoting effect of fitness qigong Wuqinxi on the rehabilitation of elderly hypertension patients," Chinese Journal of Gerontology, vol. 33, no. 7, pp. 1645-1647, 2013.

[46] Q. Lin and X. H. Yan, "Promoting effect of Baduanjin on rehabilitation of elderly hypertension patients," Chinese Journal of Gerontology, vol. 37, no. 12, pp. 3024-3026, 2017.

[47] J. Liu, "A study on the combination of Taijiquan with health management in the treatment of community patients with essential hypertension," Guiding Journal of Traditional Chinese Medicine and Pharmacy, vol. 23, no. 5, pp. 64-66, 2017.

[48] T. Liu, Q. D. Huang, and W. Z. Liu, "Effects of Taijiquan on blood pressure, hemorheology and long-term quality of life in elderly patients with hypertension," Chin J Gerontol, vol. 38, no. 6, pp. 1396-1398, 2018.

[49] W. Q. Liu, "The research of blood pressure variability and quality of life effect on patients with liver-yang hyperactivity type hypertension by Taijiquan," M. S. thesis, Nanjing University of Traditional Chinese Medicine, Nanjing, China, 2016.

[50] H. Luo, "Clinical study of Taijiquan combined with medicine in treating essential hypertension," China Medical Herald, vol. 3, no. 33, pp. 43-44, 2006.

[51] H. N. Mao and P. Sha, "Effect of Tai Chi exercise on blood pressure, plasma nitrogen monoxidum and endothelin in hypertensive patients," Chinese Journal of Clinical Rehabilitation, vol. 48, no. 10, pp. 65-67, 2006.

[52] H. S. Pan and Y. C. Feng, "Clinical observation of baduanjin exercise on the rehabilitation treatment of grade 1 hypertension in the elderly," Journal of Nanjing Institute of Physical Education, vol. 9, no. 1, pp. 4-6, 2010.

[53] A. M. Shen, Y. Wei, and J. J. Hua, "Effects of Wuqinxi on blood pressure and quality of life in elderly patients with essential hypertension stage I and II," China Journal of Chinese Materia Medica, vol. 12, pp. 2114-2115, 2010.

[54] Q. Q. Sun, "Study on the effect of Taijiquan exercise on the quality of life in elderly patients with hypertension," $\mathrm{M}$. S. thesis, Beijing Sport University, Beijing, China, 2010.

[55] Q. H. Tang, "Effects of traditional sports on clinical symptom of aged intellectual patients with essential hypertension," Journal of Beijing Sport University, vol. 32, no. 2, pp. 67-69, 2009.

[56] X. J. Wang, Y. J. Li, and N. N. Liu, "Empirical study of Taijiquan interventions on the prevention and cure of hypertension," Journal of Beijing Sport University, vol. 34, no. 09, pp. 75-77, 2011.

[57] H. Xu, "Effect of 24-style simplified Taijiquan on blood pressure and quality of life in patients with hypertension,"
Hubei Journal of Traditional Chinese Medicine, vol. 38, no. 7, pp. 38-39, 2016.

[58] D. M. Xu, "Effect of simplified Taijiquan on grade I hypertension in the elderly," China Journal of Chinese Materia Medica, vol. 11, pp. 1638-1639, 2015.

[59] H. Yang, "Effect of Baduanjin on quality of life and autonomic nervous response in patients with hypertension," M. S. thesis, Hebei United University, Hebei, China, 2014.

[60] L. W. Zheng, Q. Y. Chen, F. Chen, L. J. Mei, and J. X. Zheng, "Effects of Baduanjin exercise on vascular endothelial function in elderly patients with grade 1 hypertension," Chinese Journal of Rehabilitation Medicine, vol. 29, no. 3, pp. 223-227, 2014.

[61] D. L. Zhong, “Tai Chi for essential hypertension: efficacy and safety evaluation and mechanism research," M. S. thesis, Chengdu University of Traditional Chinese Medicine, Chengdu, China, 2019.

[62] M. Yang, L. S. Huang, X. D. Yang, J. Y. Zhuang, and Y. M. Lu, "Research on the intervention effect on people with borderline hypertension in community by baduanjin and health education," Chinese Manipulation \& Rehabilitation Medicine, vol. 4, no. 3, pp. 130-132, 2013.

[63] H. T. Zhou, "Contrastive analysis of rehabilitation effect of Tai Chi and walking on patients with hypertension," Chinese Journal of Convalescent Medicine, vol. 24, no. 5, pp. 494-495, 2015.

[64] S. W. Zhou, "Effects of Tai Ji Quan on blood pressure and lipid profile in patients with stage I essential hypertension," International Medicine and Health Guidance News, vol. 13, no. 15 , pp. 60-64, 2007.

[65] C. X. Hong, "Effect of Wuqinxi on blood pressure and C-reactive protein in patients with hypertension," World Health Digest.Medical Monthly, vol. 4, no. 8, pp. 26-27, 2007.

[66] S. Shim, B. H. Yoon, I. S. Shin, and J. M. Bae, "Network metaanalysis: application and practice using Stata," Epidemiology and health, vol. 39, Article ID e2017047, 2017.

[67] J. P. Jansen and H. Naci, "Is network meta-analysis as valid as standard pairwise meta-analysis? It all depends on the distribution of effect modifiers," BMC Medicine, vol. 11, no. 1, p. 159, 2013. 